Article Type

\title{
Machine Learning in Hazardous Building Material Management: Research Status and Applications
}

Pei-Yu Wu ${ }^{1,2,{ }^{*}}$, Kristina Mjörnell ${ }^{1,2}$, Claes Sandels ${ }^{1}$, Mikael Mangold ${ }^{1}$

1. RISE Research Institutes of Sweden, 41258 Gothenburg, Sweden; E-Mails: pei-yu.wu@ri.se; kristina.mjornell@ri.se; claes.sandels@ri.se; mikael.mangold@ri.se

2. Department of Building and Environmental Technology, Faculty of Engineering, Lund University, 22100 Lund, Sweden

* Correspondence: Pei-Yu Wu; E-Mail: pei-yu.wu@ri.se

Academic Editor: Hossein Hosseinkhani

Special Issue: $\underline{\text { New Trends on Circular Economy Building and Construction Materials }}$

Recent Progress in Materials

2021, volume 3, issue 2

doi:10.21926/rpm.2102017
Received: January 22,2021

Accepted: April 26,2021

Published: May 11,2021

\section{Abstract}

Assessment of the presence of hazardous materials in buildings is essential for improving material recyclability, increasing working safety, and lowering the risk of unforeseen cost and delay in demolition. In light of these aspects, machine learning has been viewed as a promising approach to complement environmental investigations and quantify the risk of finding hazardous materials in buildings. In view of the increasing number of related studies, this article aims to review the research status of hazardous material management and identify the potential applications of machine learning. Our exploratory study consists of a two-fold approach: science mapping and critical literature review. By evaluating the references acquired from a literature search and complementary materials, we have been able to pinpoint and discuss the research gaps and opportunities. While pilot research has been conducted in the identification of hazardous materials, source separation and collection, extensive adoption of the available machine learning methods was not found in this field. Our findings show that (1) quantification of asbestos-cement roofing is possible from the combination of remote sensing and machine learning algorithms, (2) characterization of

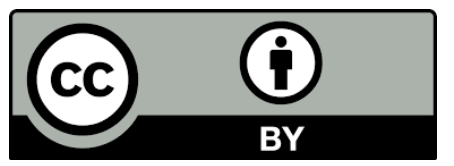

(C) 2021 by the author. This is an open access article distributed under the conditions of the Creative Commons by Attribution License, which permits unrestricted use, distribution, and reproduction in any medium or format, provided the original work is correctly cited. 
buildings with asbestos-containing materials is progressive by using statistical methods, and (3) separation and collection of asbestos-containing wastes can be addressed with a hybrid of image processing and machine learning algorithms. Analysis from this study demonstrates the method applicability and provides an orientation to the future implementation of the European Union Construction and Demolition Waste Management Protocol. Furthermore, establishing a comprehensive environmental inventory database is a key to facilitating a transition toward hazard-free circular construction.

\section{Keywords}

Machine learning technique; hazardous material management; construction and demolition waste; systematic review; asbestos-containing material; polychlorinated biphenyls (PCB)

\section{Introduction}

Hazardous building materials, such as construction products containing substances that are harmful to human health or the environment, hamper the construction and demolition (C\&D) waste management process and the quality of the recycled materials. Since C\&D waste is the largest waste stream in the European Union (EU) countries, the EU Commission developed the C\&D Waste Management Protocol and level indicators to help the member states achieve a $70 \%$ recycling rate of non-hazardous C\&D waste by 2020 [1, 2]. In the transition toward circular construction, two forces fuel the development of C\&D waste management: the push-force comes from the Waste Framework Directive 2008/98/EC, whereas the pull-force of regulating hazardous materials derives from building certification scope extension. Considering the cost and working safety of handling materials during demolition or renovation that might contain unexpected hazardous components, obligatory or voluntary pre-demolition audits on environmental inventories have been enforced in some EU countries $[1,3]$. These measures align with the actions stated in the EU C\&D Waste Management Protocol, stressing the importance of waste identification, source separation, and waste collection [4]. In addition, assessing hazardous materials and waste management in current building stock has been included in multiple sustainable building certificates worldwide, including Leadership in Energy and Environmental Design (LEED), Building Research Establishment Environmental Assessment Method (BREEAM), etc. [5]. Although countries worldwide have introduced bans on hazardous building materials for decades, harmful substances might enter the waste stream after a long life span during renovation and demolition [6]. A growing concern of disturbing residuals hinder the recyclability of the building materials exists. Therefore, the Swedish Green Building Council launched the Environmental Building Operation and Administration Certification to extend the evaluation of the existing buildings. Environmental investigations on building stock facilitate decontamination and can be instrumental for future C\&D waste management plans.

Among the hazardous materials, asbestos and polychlorinated biphenyls (PCB) are the most investigated substances because of two reasons: their critical hazardous properties and their extensive use in the middle of the $20^{\text {th }}$ century. Despite the deployment of the mandatory legal requirements, asbestos-containing materials (ACMs) and PCB-containing joints and sealants are 
diffused in the building stock, making it difficult to determine their location and amount [7]. The conventional way of quantifying in-situ hazardous materials is by conducting environmental investigations before demolition or renovation. Depending on the scale and activities in already completed buildings, environmental audits are performed for the buildings to various extents. Complex facilities, such as industrial and public buildings, often require thorough environmental investigations by qualified experts. In most surveys, laboratory analysis of samples and product labeling recognition were commonly employed by environmental experts for distinguishing harmful building materials [6]. Although the approach achieves a high accuracy rate in material identification, it is resource-demanding and time-consuming. Further, field sampling and labeling recognition are not always possible due to the inaccessibility of the building materials or the ongoing activities in buildings. Another major limitation lies in the difficulty of upscaling, leading to only a few buildings having environmental inventories. Since the information on the residual hazardous materials in the building stock is lacking, the risk of pending demolition work in local municipalities [8] and the absence of an organized waste policy of the central authority [3] remain.

Several studies have tried to develop innovative and cost-efficient methodologies to overcome the abovementioned problems. The potential of applying statistical operations in registered records $[8,9]$, creating digital tools for field inventory [10-12], etc., was explored as alternative ways for supplementing the existing methods. These novel methods successfully characterized the amount and types of ACMs in residential buildings despite the different data input and collection processes. To broaden the recognition of in-situ hazardous materials on a large scale, supervised machine learning, which enables the prediction of unknown instances based on the historical labels, presents a new opportunity to the field. The emergent machine learning techniques can identify critical features and patterns from heterogeneous data by harnessing the power of statistics, computer science, and domain knowledge. With the improved data availability and the demand for decontamination in buildings, researchers started to adopt machine learning techniques to streamline the traditional modeling process and enhance the hazardous material recognition rates. Pilot studies showed promising results in estimating the amount and the spatial distribution of insitu hazardous materials $[9,13]$ and facilitating semi-automated material sorting [14, 15] by adopting machine learning algorithms.

With regards to the call for improving the quality of C\&D waste for resource efficiency, a comprehensive literature review on the validity of various methods and their practical implementation will be beneficial. Therefore, this review article aims at examining the existing approaches and technologies to enhance the efficiency of identifying dangerous building materials. By summarizing the state-of-the-art research domains, we can pinpoint the potential applications for data-driven in-situ hazardous materials management. Accordingly, two research questions have been formulated:

RQ1: What are state-of-the-art methods for probing hazardous building materials using analytic techniques?

RQ2: What are the available applications for data-driven in-situ hazardous material management?

This study is, to our knowledge, the first review on the interdisciplinary subject of employing machine learning applications in the management of hazardous building materials. The outcomes discussed in this article can add academic value by demonstrating the hotspots and gaps in the field, 
as well as contribute to the EU C\&D Waste Management Protocol and guidelines and selective demolition ${ }^{1}$ activities in practice. This systematic literature review incorporates quantitative and qualitative dimensions. Science mapping offers an overview of the research dynamics, whereas the critical literature review analyzes the contributions of the selected references. The following section describes the search process and the structural procedure undertaken for conducting the systematic literature review.

\section{Method}

The systematic literature review was performed sequentially in two parts to measure the research dynamics in the quantitative and qualitative dimensions, as illustrated in Figure 1. In Part I, science mapping (or meta-analysis) contributes to the understanding of the domain evolution and describes the dynamics of the major disciplines [16]. The underlying structure of the research development and the conceptual and intellectual structure in hazardous materials areas could be displayed by performing a bibliometric analysis. The research scope was restricted to only in-situ hazardous materials in C\&D waste management. Therefore, off-site issues such as waste logistics, waste processing, quality management, policy and framework conditions were not included. After delineating the knowledge domain, in the critical literature review in Part II, a compilation and analysis of the highly relevant research identified in Part I has been presented. In this part, the relevant studies in which data analytic techniques have been applied in hazardous material management, i.e., hazardous material identification, waste source separation, and hazardous waste collection, have been described. The applied machine learning techniques, data input, and their purpose of use in the literature have been illustrated in the results mentioned in Part II. By associating the outcomes from science mapping and critical literature review, we can answer the research questions posed in this article.

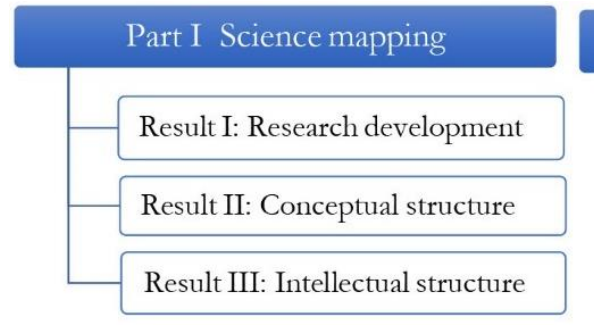

\section{Part II Critical literature review}

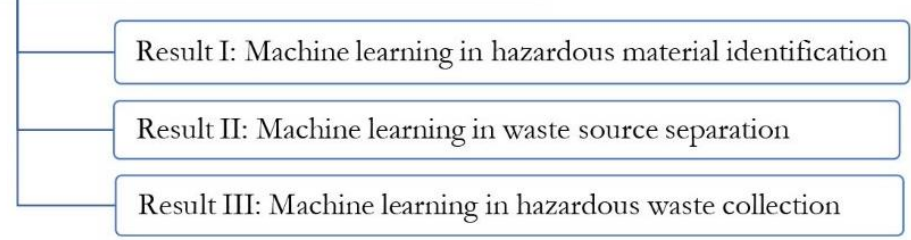

Figure 1 The comprehensive review presented in this article consists of a quantitative study (science mapping) and a qualitative study (critical literature review).

The literature search was carried out on April 22, 2020, using the Web of Sciences and Google Scholar platforms by considering the various strengths and limitations of the different algorithm capabilities. The literature available on Web of Sciences consists of publisher-neutral, peerreviewed academic papers, whereas Google Scholar provides higher adaptability in full-text searches on any type of documents. Search phrases including the words "hazard", "(artificial intelligence) Al or machine learning", and "building" topics were combined by using Boolean operators. The studies queried were English-based literature, including article, proceeding paper,

\footnotetext{
${ }^{1}$ Selective demolition means the removal of materials from a demolition site in a pre-defined sequence before demolition or renovation, in order to maximize recovery and recycling performance.
} 
review, conference paper, with the abovementioned terms in the title, abstract, author keywords, and as input to Keywords Plus ${ }^{2}$ without a specific timeframe. The preliminary search yielded four hits, and their topics were not directly relevant to the predefined search scope. Accordingly, the search was extended to incorporate synonyms of Al and machine learning, such as the words "assessment", "evaluation", "prediction", "estimation", "identification", and "modeling". Also, the types of investigated hazardous materials were specified by taking into account of their hazardous property and universality, such as radon gas from blue concrete and chemical substances from impregnated wood. The second search resulted in 436 hits, and this was further refined to exclude the irrelevant phrases or abbreviations. This iterative relevance and duplication check by reviewing the title and abstract generated 277 relevant results. As a supplement to the initial literature search, the same procedure was executed in Google Scholar, and 307 papers were finally chosen for the review. Table 1 outlines the iterative search process, search terms, and search specifications.

Table 1 Steps of the iterative search process and the combination of the search phrases used in the three topics by using Boolean operators.

Step 1: Apply search phrases in Web of Sciences and refine the irrelevant results

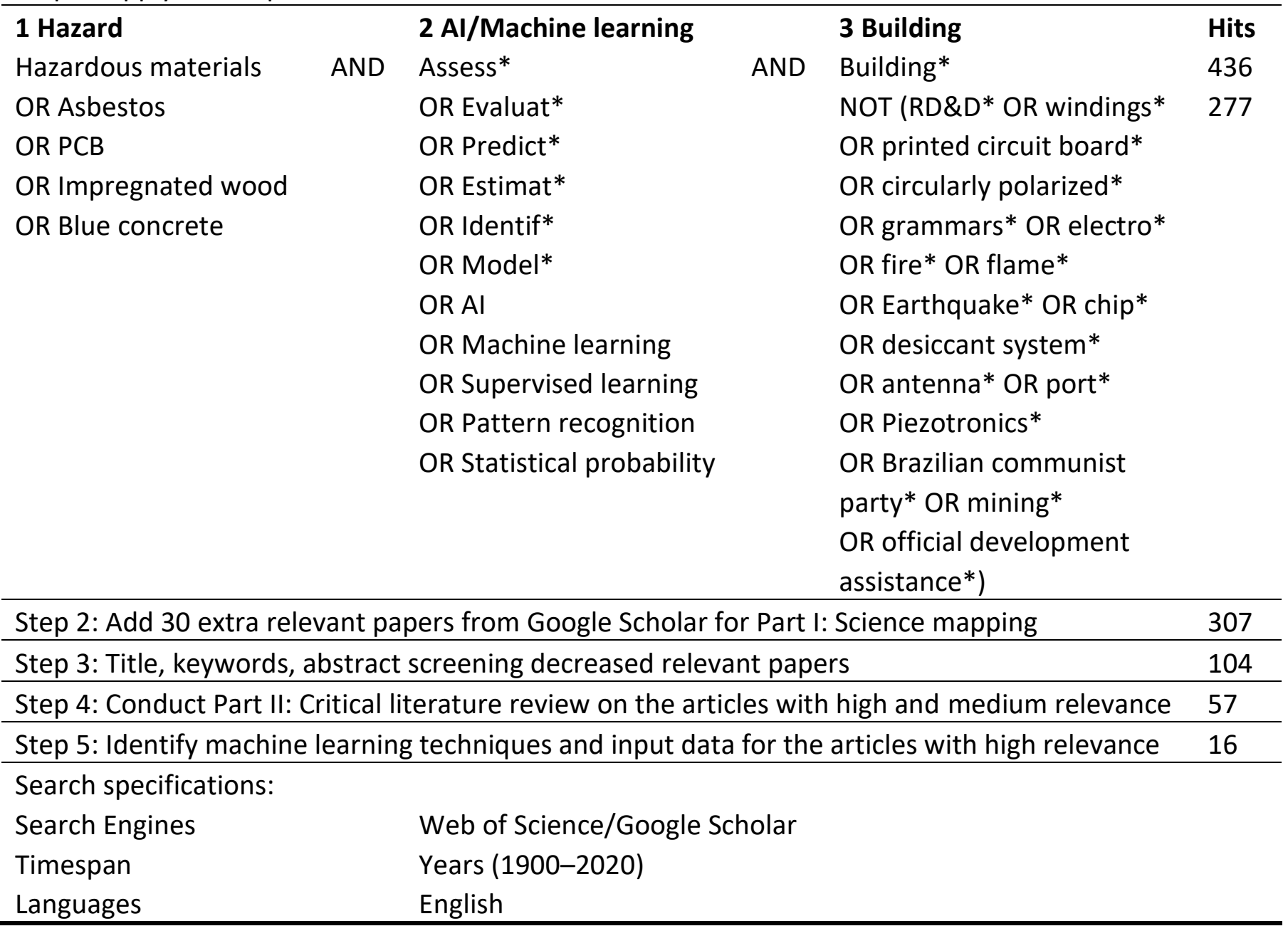

\footnotetext{
${ }^{2}$ Keywords Plus recognize phrases frequently appear in the title an article's references, but not in the article's title itself. More information about Keywords Plus:

https://support.clarivate.com/ScientificandAcademicResearch/s/article/KeyWords-Plus-generation-creation-andchanges?language $=e n$ US
} 
The asterisk $(*)$ is a type of wildcard expression. It implies that any group of characters could be combined in the search term. For example, model* will comprise model, models, modeling, etc.

Science mapping, or more specifically, bibliometric analysis, was executed on the 307 selected papers using Biblioshiny ${ }^{3}$, a bibliometric library in R. Cross comparison among the results provided insights into the thematic evolution, the collaboration of prominent authors and institutions, and the influential journals in the field of hazardous materials. Subsequently, supplementary sources from the references or citation lists and recommendations from search engines were appended manually to the document pool. Before the critical literature review, the authors performed a quick literature screening to sort out noteworthy references according to the following criteria: (i) high relevance to the concepts of hazardous materials AND Al/machine learning/quantitative methods, (ii) medium relevance to the ideas of hazardous materials $\mathrm{OR} \mathrm{Al} /$ machine learning in building materials/quantitative methods and (iii) Low relevance to the umbrella terms of $A l$ in the architecture, engineering and construction industry, Al in C\&D waste management, and Al in circular construction.

The 57 references identified with high and medium relevance were evaluated by adopting the preferred reporting items for systematic reviews and meta-analyses (PRISMA) statement, developed as an appraisal framework to minimize reporting bias [17]. Complying with the guidelines of the PRISMA statement, the following iterative procedure was adopted: (1) understanding the research problems addressed in the introduction section, (2) evaluating the effectiveness of the research procedure to its research objectives in the methodology section, and (3) reviewing the research results and their applications discussed in the discussion and conclusion section. By conducting a critical literature review, it is possible to determine the state-of-the-art research fields and research gaps.

\section{Results and Discussion}

After acquiring the literature, science mapping was performed to outline the research development and determine the significant references. We could delineate machine learning applications within the topics of hazardous material identification, waste sourcing, and hazardous waste collection through this convergent process. The identified opportunities and challenges regarding method implementation in the critical literature review have been synthesized at the end of the article.

\subsection{Science Mapping}

By evaluating the meta-data of the acquired 307 references, a full retrospect of the research on hazardous building materials in the last three decades was created. The analytical results from the research development exhibited an upward trend in interdisciplinary research activities, mainly from Environmental Sciences, Public and Environmental Health, and Engineering. Two major research themes were identified in the conceptual structure analysis: diseases and risks associated with airborne asbestos and exposure to PCB-containing materials in buildings. Significant

\footnotetext{
${ }^{3}$ Biblioshiny: https://www. bibliometrix.org/Biblioshiny.html
} 
occurrences of the terms "exposure" and "building" dominated the field. By evaluating the outcomes of the intellectual structure, a gap between the scientific research and management of hazardous building materials in the field of C\&D waste was recognized. Despite the growing number of studies addressed toward exposure measurement and risk remediation of harmful substances in buildings, very few can be integrated into the context of in-situ hazardous material management.

\subsubsection{Literature Distribution}

The first bibliometric analysis performed in this work measured the research developments in hazardous material management by characterizing the annual scientific production, thematic distribution, and the publication outlet for the references included in this study. The combined search resulted in 307 references from 188 sources, including books, journals, and thesis publications. Studies in this area were published sporadically during a longer timeframe. However, an upward trend was observed since the 1990s with a publication peak in 2016-2018, as shown in Figure 2. Over the publication period, the geometric progression ratio was $5.95 \%$, a constant quantity by which each scale factor in a geometry progression was multiplied to be the succeeding ones [18]. It indicates the average increasing rate of papers concerning the accumulated sum of the published articles. In addition, $70 \%$ of the references were published in the last 15 years. A possible explanation for this might be that the awareness of hazardous building materials was first raised after international bans and restrictions imposed since the mid-1970s. During the 1990s, countries worldwide introduced extensive bans on ACMs in terms of production, import, and sales [19], which coincides with the growing development of the field and the increase in the number of relevant research activities.
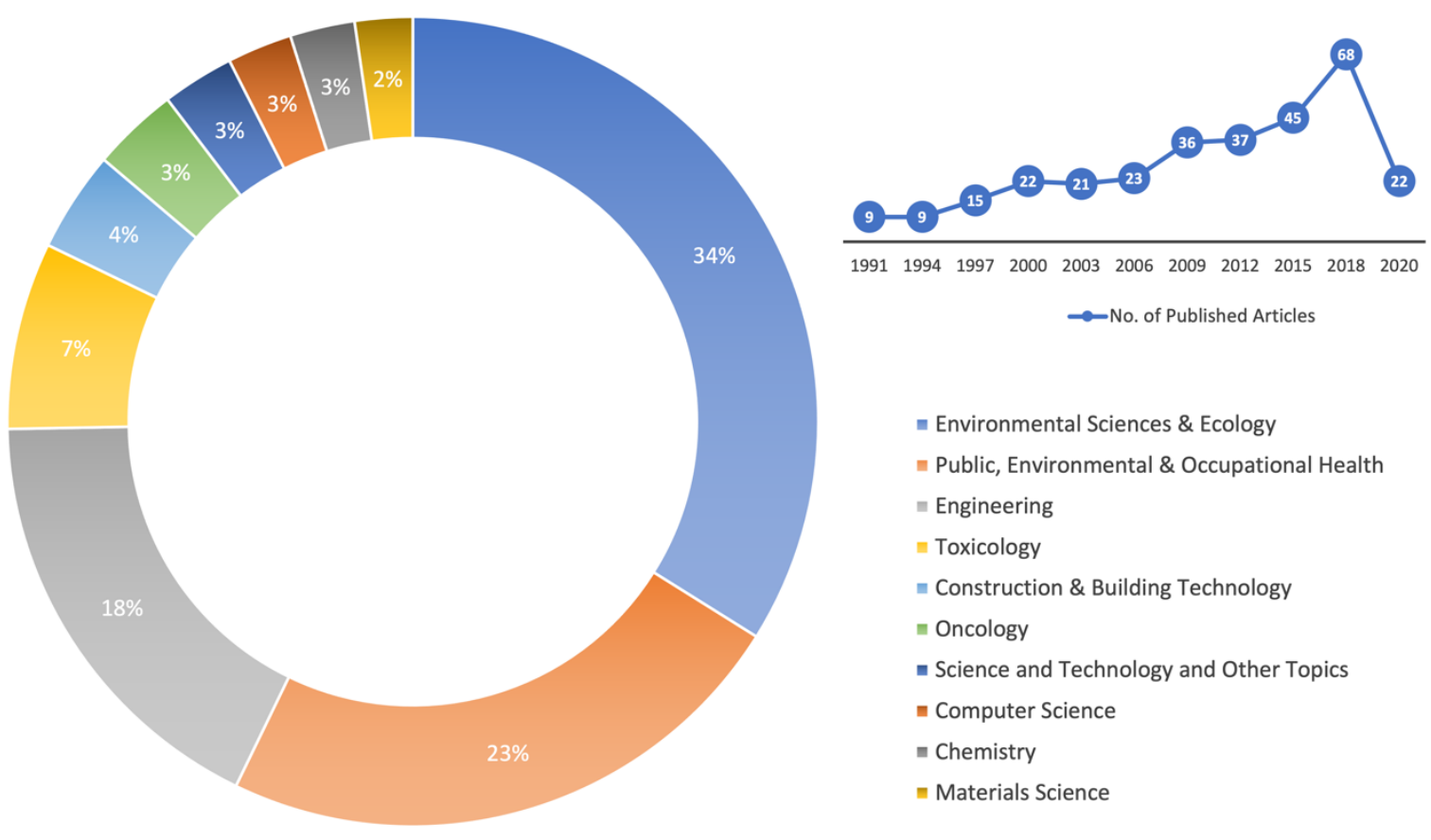
Figure 2 Measurement of the research development by quantifying the annual scientific publications from 1991-2020 (top right) and evaluation of the thematic distribution of the major source publications (bottom left).

Other than evaluating the number of publications, the thematic distribution of the source publications indicates the progress of multi-disciplinary studies. Figure 2 highlights a dispersed, uneven thematic composition of the source publications from the acquired references. The thematic categories have been listed in descending order according to their proportion. A large number of source publications were categorized to "Environmental Sciences \& Ecology", "Public, Environmental \& Occupational Health", and "Engineering" according to the Web of Science platform. This result indicates the interdisciplinary characteristics of these subjects with general interests in quantifying environmental and health-related issues.

On the other hand, only a handful of papers were published in "Construction \& Building Technology" related journals, representing $4 \%$ of the total selected references. According to source clustering, they were primarily published in Environmental Science \& Technology, Chemosphere, International Science and Pollutant Research, Indoor and Built Environment, and Journal of Hazardous Materials. As a significant number of source publications belong to environmental and chemical studies, it was not surprising to see research related to sample monitoring of hazardous substances [21, 22], their mitigation and remediation measures [23], and their risk management [24]. In the thematic distribution of the publication journals, the references that involved machine learning concepts were selected before proceeding to the critical literature review.

\subsubsection{Conceptual Structure}

The conceptual structure benchmarked the research front and domain evolution to identify the essential issues in the area, represented in the co-word analysis and word dynamic analysis. The coword analysis shown in Figure 3 assembles the most contributing papers and associates similar variable categories using multivariate statistics. The multiple correspondence analysis (MCA), a modified principal component analysis method used for detecting and representing the underlying structures in a nominal categorical dataset, was applied to the references to identify groups of words obtained using Keyword Plus with similar profiles and the correlations between the variable categories. The results from Keyword Plus were represented as data points in factorial planes (or Euclidean planes) after dimensionality reduction. The axis corresponds to the first and second principal components, and the percentage accounted for the amount of variance [25]. Correlations between the results obtained from Keyword Plus were uncovered by calculating the proportional variance between the categorical variables of Keyword Plus and the individual reference [26]. The findings indicate that research on asbestos and PCB-related subjects constitutes the most knowledge base in the acquired literature. In contrast, keywords related to impregnated wood and blue concrete were missing due to insufficient studies. Based on their positions on the two dimensions in Figure 3, the variances between the umbrella terms of asbestos (the red group) are less significant than those of PCB (the blue group). For example, the keyword asbestos and its synonyms ${ }^{4}$ were associated closely with "occupational exposure", "lung cancer and mesothelioma",

\footnotetext{
${ }^{4}$ Asbestos has different forms in construction products, such as chrysotile, amosite, crocidolite. All of them can cause asbestosis, mesothelioma, and lung cancer on high levels of exposure.
} 
and "mortality risk" in one cluster. On the other hand, the keyword PCB was related to the studies on "indoor air contamination" and "congeners exposure" in another cluster.

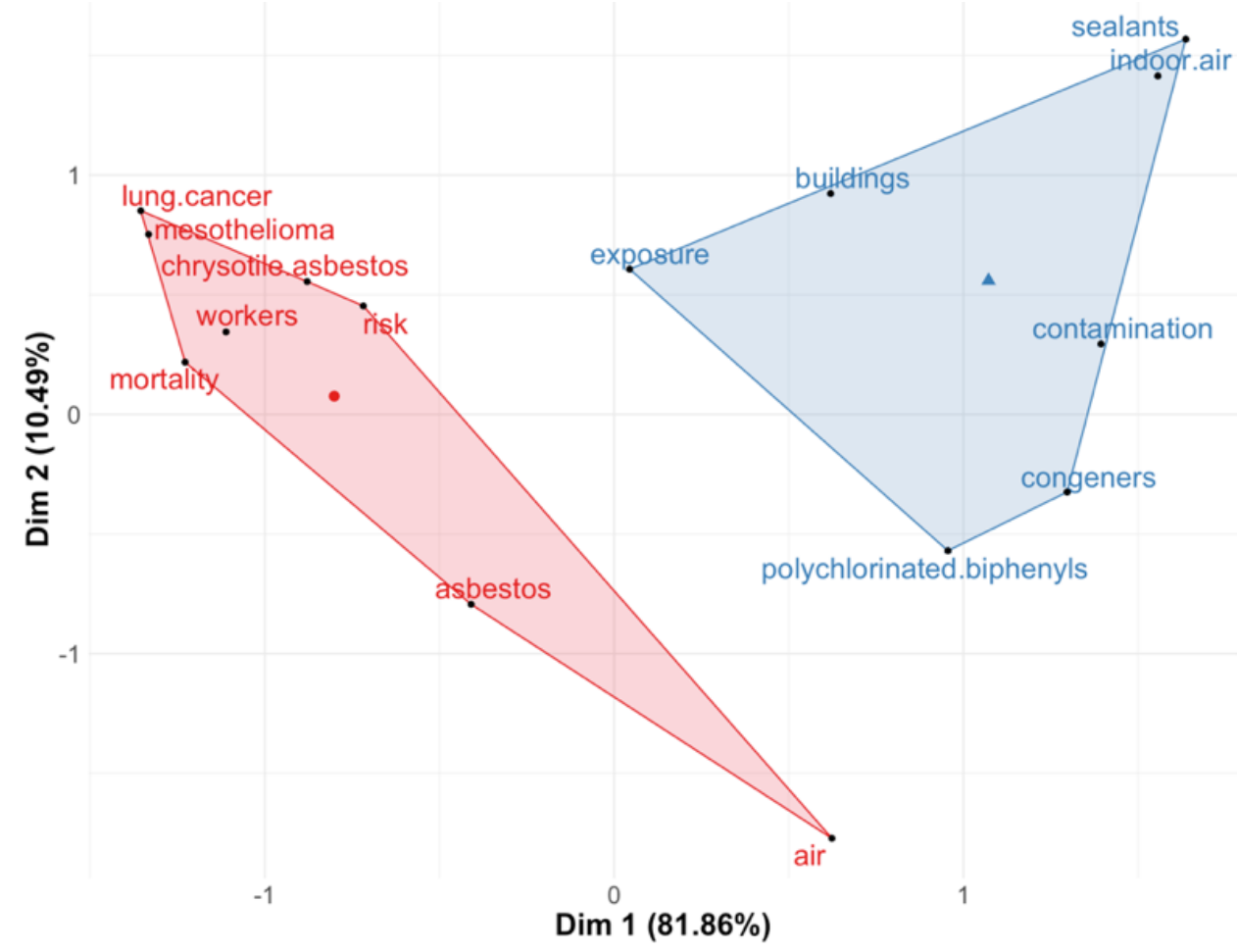

Figure 3 The co-word analysis measures the similarity and dissimilarity between the generated Keyword Plus from the acquired references using the MCA method. Dimension 1 (81.86\% explain the variance) covers the opposition between the asbestos cluster and the PCB cluster. Dimension 2 (10.49\% explain the variance) includes the opposition within each cluster.

On the contrary, references corresponding to impregnated wood and blue concrete are relatively rare. The fewer articles available on these topics might be due to their low presence in buildings compared to asbestos and PCB, leading to very few quantitative studies on them. This discrepancy can also be attributed to the legislative framework, where asbestos and PCB have their regulations and initiatives in the Nordic countries [7]. Furthermore, the word dynamic analysis shown in Figure 4 presents a divergent tendency of the top 10 research terms cumulated in the past decades. By creating an overview of the various timespan, the results indicate the evolved topics. The primary research focus is on the working safety and pollutant exposure to asbestos and PCB in the built environment. However, the research terms exposure and building experienced explosive growth since 2012. Surprisingly, terminologies of hazardous material management, such as risk assessment, identification management, working modeling, etc., were less studied subjects. This might explain why the literature inquiry to the quantitative research using machine learning techniques yielded no distinguished hits since the interdisciplinary topic is still in the embryonic phase. 


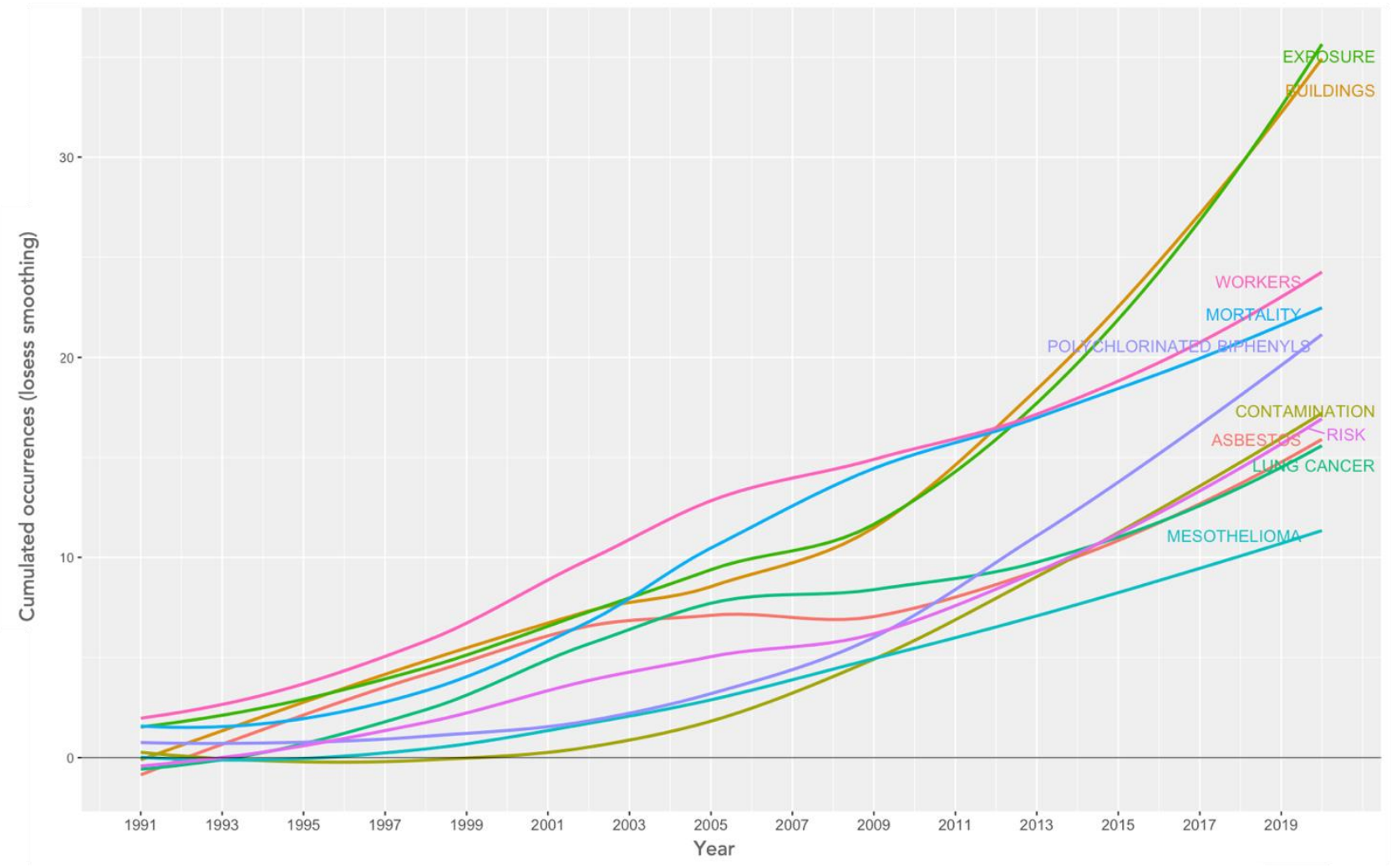

Figure 4 Word dynamic analysis. The cumulated occurrences of the generated Keyword Plus present the shift in the research terms in the last three decades. The top frequent terms are identified as exposure, buildings, workers, mortality, polychlorinated biphenyls, contamination, risk, asbestos, lung cancer, and mesothelioma.

\subsubsection{Intellectual Structure}

The intellectual structure demonstrates the paradigm shift in a given knowledge domain, showing the citation relationship between the references [27]. It involves a historical direct citation network and a three-field plot. The historical direct citation network was presented in the format of historiographic mapping as it provided a general picture of the research topics with respect to the citation of core authors and documents. Five primary research paths were identified that are presented in Figure 5 with different colors: (1) asbestos concentrations and abatement, (2) asbestos-related diseases, (3) pollution and health, (4) asbestos investigation, and (5) PCB sources and emissions. To summarize the science mapping section, a three-field plot was created, as shown in Figure 6, to determine the correlation between the author's keywords, authors, and publication journals. The findings show that the included references were mainly published in the journals related to environmental science, such as Environmental Science and Technology, Indoor and Built Environment, Environmental Science, and Pollution Research, with various focus areas, in particular, environmental health, hygiene, and medicine. It suggested that the management of the in-situ hazardous materials and their risk remediation were studied more in relation to environmental sciences than in waste management. The critical literature review in the next section explores the potential synergies between the two disciplines. 


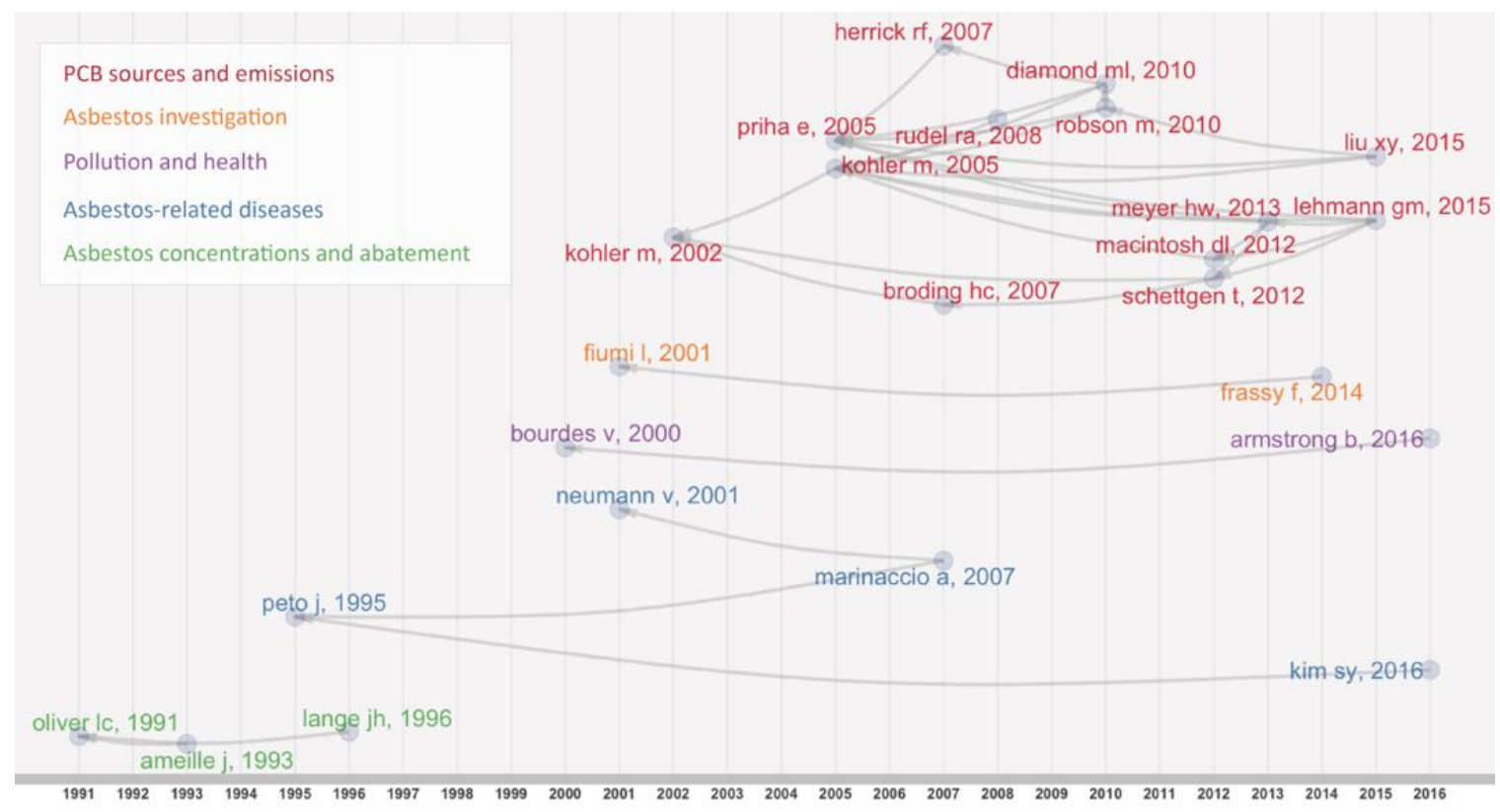

Figure 5 Historical direct citation network. The citation links by year of publication have been categorized into five thematic groups represented in the legends.

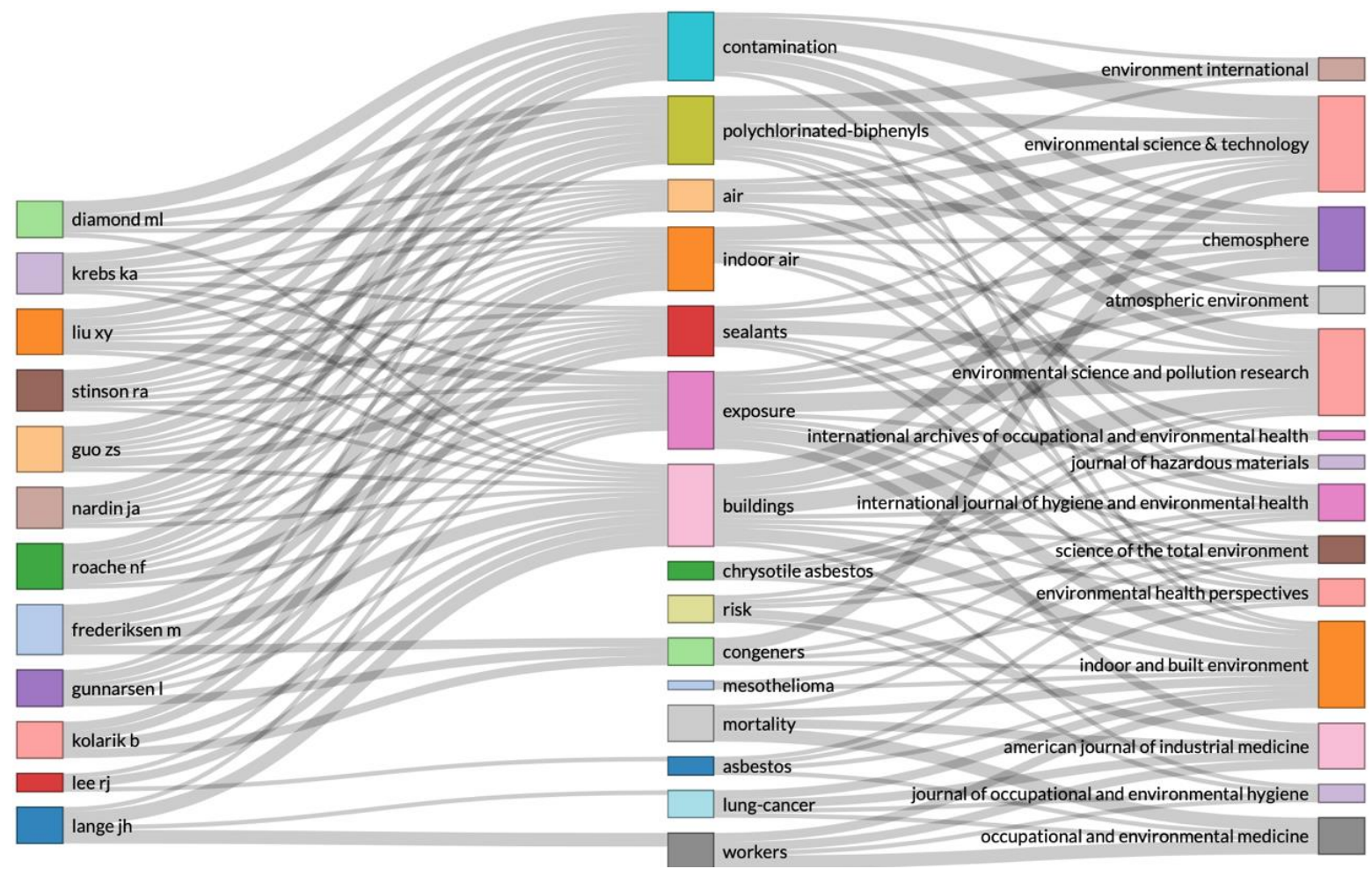

Figure 6 Three-fields plot. The most trending author's keyword (middle) associate with the most contributing authors (left) and the frequent publication journals (right).

\subsection{Critical Literature Review}

Growing concern has been expressed within the construction sector [28] about the presence of hazardous materials in building products, which hampers circular construction. Thus, various studies have tried to address the issue of risk management of hazardous substances in the circular 
construction value chains and the health and safety aspects of the building stock. Novel tools and assessment schemes have been developed for quality control of the C\&D waste to minimize the use of recycled materials containing hazardous substances [7]. Continuous research for managing hazardous residuals in the built environment was found in local contexts. For instance, Donovan and Pickin [29] modeled the stock and flow for asbestos metabolism in Australia, Kim and Hong [30] proposed safety management and disposal programs for ACMs in extreme conditions in Korea, etc. Eventually, the application domains of the 57 references included in the critical literature review were mapped out in Table 2.

Table 2 Categorization of the research domains in the critical literature review $(N=57)$.

\begin{tabular}{lll}
\hline Research domain & No. & References \\
\hline Machine learning in hazardous material identification & 9 & {$[8-13,31-33]$} \\
Machine learning in source separation & 2 & {$[15,34]$} \\
Machine learning in hazardous waste collection & 5 & {$[14,35-38]$} \\
Hazardous building materials & 3 & {$[5,28,39]$} \\
C\&D waste management & 5 & {$[2-4,6,7]$} \\
Asbestos in buildings and related diseases & 14 & {$[24,40-52]$} \\
PCB in buildings & 19 & {$[21,23,53-69]$} \\
\hline
\end{tabular}

Although different quantification approaches were developed, a fundamental challenge in the risk management of hazardous materials has remained in the disconnected legal frameworks [28]. To resolve the structural problem and harmonize separate guidelines, the EU C\&D Waste Management Protocol and Guidelines were established for improving material identification, separation, and collection at the source [4]. The protocol has enabled effective in-situ and off-site hazardous material management in the early stage of C\&D waste processing. Therefore, to pinpoint the significant findings of previous studies within a larger framework, highly relevant studies using data analytic techniques were organized following the first part of the objectives of the protocol. Figure 7 presents an overview of the available applied machine learning applications specifically for data-driven in-situ hazardous material management. Correspondingly, a summary of the critical references has been presented in Appendix $A$, and their major contributions to the field have been compiled in Appendix B. 


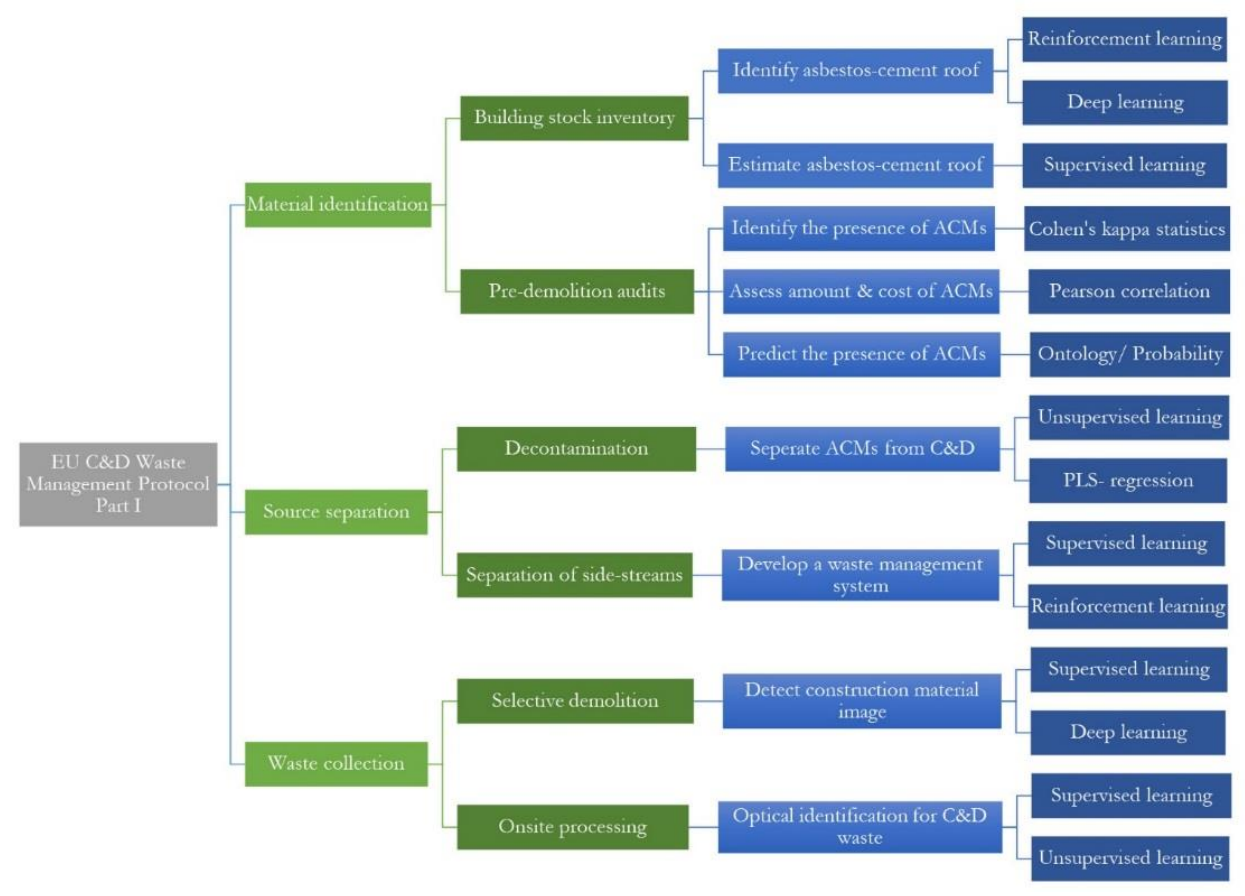

Figure 7 Overview of the research applications of the corresponding activity flow in the EU C\&D Waste Management Protocol Part I [4]. In-situ hazardous material management (light green, level 1) comprises of multiple sub-tasks (dark green, level 2) that can be complemented by adopting the data-driven applications (light blue, level 3) using applied machine learning techniques (dark blue, level 4).

According to our analysis, the broad concepts of applied machine learning have been adopted for hazardous material detection, assessment, estimation, and prediction in the previous studies. Algorithms and statistical methods were employed for quantifying the hazardous materials in the regional building stock and individual buildings. Mapping the applied machine learning applications in the present study can not only add to the current C\&D waste practice but also advance the longterm building stock research as a whole. By associating the research purpose and the corresponding machine learning techniques in the acquired literature, we have obtained an overarching picture of the state-of-the-art research potential and limitations.

\subsubsection{Machine Learning in Hazardous Material Identification}

Waste identification requires a clear definition of waste and a thorough environmental inventory attained from pre-demolition audits (or environmental audits) and waste management plans [4]. The environmental inventories provide critical information about the amount and location of the hazardous materials, thus increasing safe waste handling by preventing second contamination [6]. In addition, they are regarded as a necessary document for selective demolition and on-site waste sorting [39]. Therefore, most studies have focused on developing methods for tracing ACMs on different scales. Our findings show a trend, based on which other data sources and formats can be combined to achieve this goal. For instance, generic data, such as property registers, historical data, geographic information system data, etc., and specific data, such as environmental inventory, description of ACMs, hyperspectral data, etc., are the essential data inputs for machine learning modeling and statistical analysis. Pre-studies on how to manipulate these data to quantify and 
characterize asbestos were recognized from the literature. However, using them as labels to train machine learning classifiers comes in a later research stage. In this sense, previous studies contributed an excellent foundation for research by establishing databases and building experience in operating multiple data types.

Research related to statistical learning, presented in Table 3, for in-situ waste identification can be approached from a macro- and a micro-perspective. The macro-level involves detection and estimation of regional ACMs via image recognition in remote sensing. By compiling the aerial photographs, hyperspectral data, and multispectral imagery, Krówczyńska et al. [13] built a hybrid database for modeling the quantity and location of asbestos-cement roofing. In their findings, the convolutional neural networks exhibited an overall high accuracy in classifying nature color images and color-infrared imagery of asbestos-cement roofing from other roof materials. Apart from employing the deep learning algorithm, supervised machine learning has also achieved promising results in modeling the spatial distribution of asbestos-cement products. According to the study by Wilk et al. [9], the Boruta algorithm extracted features effectively from a pool of diverse datasets for feature extraction in data preprocessing, including statistical features, building-up areas, field inventory, and historical information on asbestos manufacturing plants. By applying these features to the random forest (RF) classifier, a satisfactory accuracy rate for mapping the nationwide spatial distribution of the amounts of ACMs could be achieved. Their results suggest that the approach is transferable for other EU countries to uptake the data on the built environment for predicting insitu asbestos.

Table 3 Summary of the statistical learning techniques applied in hazardous material identification.

\begin{tabular}{|c|c|c|c|}
\hline Purpose of use & Techniques & Data specifications & References \\
\hline $\begin{array}{l}\text { Identify asbestos-cement } \\
\text { roofing }\end{array}$ & R-Keras, CNNs & $\begin{array}{l}\text { Aerial images, hyperspectral } \\
\text { data, multispectral images }\end{array}$ & [13] \\
\hline $\begin{array}{l}\text { Mapping asbestos- } \\
\text { cement roofing }\end{array}$ & Boruta algorithm, RF & $\begin{array}{l}\text { Statistical features, built-up } \\
\text { areas, field inventory, } \\
\text { historical data }\end{array}$ & [9] \\
\hline Evaluate classifiers & LDFA, QDFA, RF & WorldView-2 images & [32] \\
\hline $\begin{array}{l}\text { performance in mapping } \\
\text { asbestos-cement roofing }\end{array}$ & Bayes, k-NN, SVM, RF & WorldView-2 images & [33] \\
\hline $\begin{array}{l}\text { Identify the presence of } \\
\text { ACMs }\end{array}$ & $\begin{array}{l}\text { Cohen's kappa } \\
\text { statistics }\end{array}$ & $\begin{array}{l}\text { Mobile app questionnaire, } \\
\text { asbestos survey }\end{array}$ & [10-12] \\
\hline $\begin{array}{l}\text { Assess amount and cost } \\
\text { of ACMs }\end{array}$ & $\begin{array}{l}\text { Scatterplots, } \\
\text { histograms, Pearson } \\
\text { correlation }\end{array}$ & $\begin{array}{l}\text { Building registers, pre- } \\
\text { demolition audits }\end{array}$ & [8] \\
\hline $\begin{array}{l}\text { Predict presence of } \\
\text { ACMs }\end{array}$ & $\begin{array}{l}\text { Ontology-based } \\
\text { approach, } \\
\text { probabilities }\end{array}$ & $\begin{array}{l}\text { Approved type project, } \\
\text { asbestos diagnosis, ACM } \\
\text { products }\end{array}$ & {$[31]$} \\
\hline
\end{tabular}

* Abbreviation of classifiers: Convolutional neural networks (CNNs); Linear/Quadratic discriminant function analysis (LDFA/QDFA); Random forest (RF); k-nearest neighbor (k-NN); Support vector machine (SVM) 
Furthermore, researchers explored various image preprocessing techniques and compared the performance of different classifiers to improve their detection accuracy [32]. For instance, Taguchi optimization techniques were used for segmenting heterogeneous urban areas before conducting an object-based image analysis. In differentiating roof-class and urban features, the RF classifier was found to be superior to the other supervised classifiers, such as Bayes and support vector machine (SVM), and unsupervised classifiers, such as k-nearest neighbor (KNN) [33]. The results were in agreement with the study by Abriha et al. [32], where pan-sharpened imagery was used for discriminant function analysis (DFA). Even though linear DFA (LDFA) and quadratic DFA (QDFA) helped in classifying the roof class, the accuracy of the RF classifiers outperformed both, in the three- and six-class. Despite the various image preprocessing techniques and analyzed methods, the two research results are comparable for the same image type and study object. To summarize, previous research studies have gained promising progress in developing practical approaches for monitoring and quantifying the presence of ACMs with the help of machine learning, which could support the authorities to devise an abatement policy and remediation strategies for hazardous materials.

Contrary to asbestos detection on a large scale, no research was found where a machine learning model has been applied directly on the micro-scale. However, statistical learning and the relevant techniques are observed in statistics to determine in-situ ACMs in a few inference studies. In a case study at the city level, Pearson correlation and visual analytics were applied to building registers and demolition datasets for characterizing ACMs in abandoned residential dwellings. According to Franzblau et al. [8], this approach successfully described the extent and the types of ACMs, and also the related remediation costs in abandoned residential dwellings. A similar study in assessing ACMs in a residential environment was conducted by Govorko et al. [10] using a different data collection approach. Rather than merging the existing datasets from the municipality, they developed a mobile application to collect information on in-situ ACMs from the property owners. The self-assessment questionnaire results were validated by performing accuracy tests and agreement with expert investigations before analyzing Cohen's kappa statistics [11]. Their findings highlighted the frequent presence of asbestos (82.3\%) in the residential environment [12], not far from the results (95\%) of the study by Franzblau et al. [8]. Both the top-down and bottom-up research methods identified prevalent types of ACMs and prioritized remediation in residence. Depending on the sample age and dwelling type, results varied in regional contexts. However, the research outcomes can be used as data labels in the future for supervised machine learning prediction. Beyond the conventional sampling methods, Mecharnia et al. [31] introduced an ontology-based approach to calculate the possibility of asbestos presence in buildings based on the temporal data. The limitations of this study are the insufficient number of variables and a lack of other relevant references for comparison. However, their inference study achieved a satisfactory result in real datasets and demonstrated correlation among other parameters. Overall, the statistical elements of these studies contribute to understanding the underlying pattern of ACMs in the characteristics of buildings, components, and products. The developed methodology can be used for developing machine learning models in the future.

\subsubsection{Machine Learning in Waste Source Separation}


Source separation is essential for eliminating hazardous waste by decontaminating it and splitting the side-streams $[4,6]$. In the case of ACMs, the requirements for the safety program and decontamination were investigated in vermiculite attic insulation in residential premises [70], vinyl flooring, asbestos-cement products [47], and asbestos insulation boards in schools [22]. Similarly, PCB contamination was found extensively in caulk and sealants in school buildings [21]. On-site investigations are standard practice for planning decontamination and locating the side-streams before demolition. However, they have limitations and can only be implemented on a small scale because of their resource-demanding, time-consuming, and circumstance-specific nature. Machine learning models for material recognition can be used for some of these works and can enable efficient source separation. The relevant research studies on this aspect have been presented in Table 4.

Table 4 Summary of the machine learning techniques applied in waste source separation.

\begin{tabular}{llll}
\hline Purpose of use & Techniques & Data specifications & References \\
\hline $\begin{array}{l}\text { Discriminate ACMs from the } \\
\text { C\&D material }\end{array}$ & PCA, PLS-DA & $\begin{array}{l}\text { Hyperspectral minerals } \\
\text { images }\end{array}$ & [15] \\
ACMs detection & PCA, SIMCA & $\begin{array}{l}\text { Hyperspectral minerals } \\
\text { images }\end{array}$ & [34] \\
\hline
\end{tabular}

* Abbreviation of classifiers: Principal Component Analysis (PCA); Partial Least-SquareDiscriminant Analysis (PLS-DA); Soft Independent Modeling of Class Analogies (SIMCA)

Effective separation of hazardous materials helps in dividing the mixed C\&D waste and increases their recyclability. Researchers explored the potential of image data and various types of waste data for training supervised, unsupervised, and reinforcement machine learning. In a study by Bonifazi et al. [34], an innovative approach based on hyperspectral imaging (HSI) was explored for detecting and classifying asbestos fibers. By combining the method of principal component analysis (PCA) and soft independent modeling of class analogies (SIMCA), it was possible to differentiate the different types of asbestos fibers according to their chemometrics, i.e., amosite, crocidolite, and chrysotile. Building upon the result, in a subsequent study by Bonifazi et al. [15], PCA and hierarchical partial least-square-discriminant analysis (PLS-DA) were further applied to discriminate the ACMs from the rest of the C\&D waste flow stream. Their work achieved non-destructive recognition of ACMs in C\&D wastes. Further research is required to evaluate the accuracy and granularity of the HSI approach for extensive implementation at the industrial level.

\subsubsection{Machine Learning in Hazardous Waste Collection}

Hazardous materials cannot be separated entirely as these dangerous substances might retransfer from the secondary contaminants, which are polluted from the primary pollutants [6]. Therefore, it is necessary to conduct selective demolition and on-site processing to collect hazardous wastes before destruction [4]. Fueled by the economic incentives in the C\&D waste market and the legislative obligations, a tendency toward semi-selective demolition ${ }^{5}$ has been observed. However, a study by Bergmans et al. [71] expressed concerns about the low quality,

\footnotetext{
${ }^{5}$ Semi-selective demolition is a demolition work where the demolition company selectively collects all hazardous substances and the part of the non-hazardous substances that would overly reduce the quality of the stony fraction.
} 
incomplete environmental inventory, and insufficient knowledge on the potential presence of hazardous materials that could impede the process of selective demolition. Thus, new tracing systems for C\&D waste quality management were developed and tested in Flanders [71]. Novel techniques for optical identification of C\&D wastes were developed by Anding et al. [37, 38]. A high potential was demonstrated for developing on-site waste management systems by employing supervised training and reinforcement machine learning models using the images of the recycled materials and aggregates. A summary of the major machine learning applications in this sub-domain using image processing is listed in Table 5.

Table 5 Summary of the machine learning techniques applied in hazardous waste collection.

\begin{tabular}{|c|c|c|c|}
\hline Purpose of use & Techniques & Data specifications & References \\
\hline $\begin{array}{l}\text { Develop a waste } \\
\text { management system }\end{array}$ & CNNs, SVM & Material images & {$[14]$} \\
\hline $\begin{array}{l}\text { Detect construction } \\
\text { material images }\end{array}$ & $M L P, R B F, S V M$ & Material images & [35] \\
\hline \multirow[t]{2}{*}{$\begin{array}{l}\text { Optical identification for } \\
\text { the C\&D waste }\end{array}$} & $\begin{array}{l}\text { PCA, LibSVM, RF, k-NN, } \\
\text { J48 Tree, Naive Bayes }\end{array}$ & $\begin{array}{l}\text { Material \& mineral } \\
\text { aggregate images }\end{array}$ & {$[37,38]$} \\
\hline & $\begin{array}{l}\text { k-NN, C-SVM, Nu-SVM, } \\
\text { Naive Bayes, RF, J48 Tree }\end{array}$ & $\begin{array}{l}\text { Material images in VIS } \\
\text { and IR spectrum }\end{array}$ & [36] \\
\hline
\end{tabular}

* Abbreviation of classifiers: Random forest (RF); k-nearest neighbor (k-NN); Support vector machine (SVM)); Principal Component Analysis (PCA); Multilayer Perceptron (MLP); Radial Basis Function (RBF); Convolutional neural networks (CNNs)

These studies explored the application of image processing of materials and minerals due to their favorable outcome in hazardous waste collection. Among all the supervised machine learning models, in the study by Kuritcyn et al. [36], the RF and SVM classifiers achieved high total recognition rates in classifying images of recycled materials and detecting materials under visible (VIS) and infrared (IR) spectrum. This result was also accorded with the corresponding results obtained by Adedeji and Wang [14], Rashidi et al. [35], and Anding et al. [38], where a combination of deep learning and supervised and unsupervised machine learning was employed. Despite the excellent performance of the presently available algorithms in recognizing hazardous materials, Anding et al. [37] suggested further improvement for detecting the digital images of mineral aggregates. The low identification rate can be attributed to the high phenotypical object variabilities within the subclasses. Tese studies open the possibility of automatic optical identification of C\&D waste and provide advantages of saving time and cost. Future works are suggested to assess the feasibility of adopting intelligent waste collection in practice and potential challenges.

\subsubsection{Opportunities and Risks of Adopting Applied Machine Learning}

Previous studies that applied machine learning in ACM identification, separation, and collection have achieved good progress. They have proved the potential of using multiple data inputs to train supervised machine learning classifiers. Their results showed that high-quality data, adequate sample size, and the use of domain knowledge are the key factors that lead to high prediction rates. 
Hence, establishing comprehensive databases for compiling the information of buildings and their environmental records are prerequisites for enabling data-driven hazardous material management. Additional empirical studies are required for addressing the risk of lacking patterns, which is still unclear when scaling up the research to the building stock level and extending the data scope to multiple hazardous materials. Further, to bridge the gap between theoretical and practical implementation, it is necessary to consider the stakeholders' perspectives when determining the subjects for prediction. By creating a deeper understanding of the potential opportunities and risks of applied machine learning, researchers and practitioners in the relevant domains can make a joint effort to expedite long-term progress in circular construction.

\section{Conclusions}

The rapid development of C\&D waste management has been shaped by the tightened legislation for circular construction and extending the assessment for a healthy building stock in certification. This is because very large amounts of ACMs and PCB-containing materials have diffused into the built environment from the middle of the $20^{\text {th }}$ century, leading to extreme difficulty in the decontamination of the building environment. Therefore, researchers have tried to develop datadriven approaches to improve hazardous material management. The purpose of this paper is to review the up-to-date quantitative studies and identify the research gaps and implementation opportunities. The deficiency of the relevant literature has indicated that only a few studies have been conducted on this emergent subject. The science mapping findings have also suggested an interdisciplinary research field based on the wide distribution of the publication journals and their scopes. In the case of building-related publication, remediation and risk assessment for asbestos and PCB were observed to be the most addressed topics, whereas hazardous materials in C\&D waste were studied as a minor stream. This fact signifies that the research of in-situ hazardous material management and demolition processing is not integrated. Further, despite the understanding of the dangerous properties of these materials and the advanced techniques developed for monitoring them, investigation of hazardous materials is still restricted to a limited scale. Difficulty in their visual recognition, lack of regulatory mandates, costly and time-consuming laboratory sampling are a few attributes that explain the stagnant development in this field. The introduction of new investigation approaches to exploit the existing environmental information effectively can provide an alternative solution.

Machine learning is a promising method that can supplement environmental investigations due to its power of predicting unknown instances based on historical records. In the critical literature review, we analyzed the various applied machine learning techniques and data input with respect to hazardous material identification, separation, and collection. This analysis has shown that a combination of algorithms and preprocessing techniques achieve high accuracy in distinguishing ACMs without intrusive sampling. Supervised machine learning was, foremost, the most explored subarea, and the RF and SVM classifiers vector machine perform the best in most cases. Mapping and quantifying $\mathrm{ACM}$ s on the regional scale can be achieved via remote sensing, whereas characterization and identification of buildings contaminated by asbestos can be attained using statistical techniques. Although studies on the building environment have not yet reached the machine learning level, their results offer valuable insights for feature selection. 
On the other hand, hazardous waste separation and collection can be realized by employing image processing for separating the ACMs in C\&D wastes. Most studies have adopted imagery data to train the classifiers, whereas the other research direction exploits the established databases. Our work contributes to an in-depth overview of machine learning applications in hazardous material management and facilitates the implementation of the EU C\&D Waste Management Protocol. Even though applied machine learning research in the field of hazardous material is still in the early stage, great potential has been demonstrated, and further practical implementation awaits to be explored.

\section{Acknowledgments}

The work is financed by the project fund for the prediction of hazardous materials in buildings using Al (FID18-0021) from the Swedish Foundation for Strategic Research and supported by the Research Institutes of Sweden.

\section{Additional Materials}

The following additional materials are uploaded at the page of this paper.

1. Table S1: Summary of the references using machine learning techniques from the critical literature review $(\mathrm{N}=16)$.

2. Table S2: Main contributions of the references using machine learning techniques from the critical literature review $(\mathrm{N}=16)$.

\section{Author Contributions}

Claes Sandels conceived the idea of conducted the literature review of machine learning application in hazardous building material field. Pei-Yu Wu and Kristina Mjörnell performed the literature search together. Pei-Yu Wu executed the science mapping and the critical literature review and drafting the initial manuscript. All of the authors participated in results discussion and manuscript revision. Mikael Mangold provided suggestions for answering the reviewers and helped disseminate the research outcome. Proofreading was performed by Pei-Yu Wu and Kristina Mjörnell, who is responsible for the project management and fund acquisition.

\section{Funding}

The Swedish Foundation for Strategic Research funds the four-year PhD project.

\section{Competing Interests}

The authors declare there is no conflict of interest in the reported work.

\section{References}

1. Dodd N, Donatello S. Level(s) indicator 2.2: Construction and demolition waste and materials user manual: Overview, guidance and instructions (publication version 1.0) [Internet]. Brussels: European Commission; 2020 [cited 2021 January 17th]. Available from: https://susproc.jrc.ec.europa.eu/product-bureau/sites/default/files/2020- 
10/20201013\%20New\%20Level(s)\%20documentation 2.2\%20C\&d\%20waste Publication\%20 v1.0.pdf.

2. Akbarieh A, Jayasinghe LB, Waldmann D, Teferle FN. BIM-based end-of-lifecycle decision making and digital deconstruction: Literature review. Sustainability. 2020; 12: 2670.

3. Wolff G. Resource efficient use of mixed wastes improving management of construction and demolition waste [Internet]. Brussels: European Union; 2017 [cited 2021 January 17th]. Available from: https://op.europa.eu/en/publication-detail/-/publication/78e42e6c-d8a611e7-a506-01aa75ed71a1/language-en.

4. ECORYS. EU construction \& demolition waste management protocol [Internet]. Brussels: European Commission; 2016. Available from: https://ec.europa.eu/growth/content/euconstruction-and-demolition-waste-protocol-0 en.

5. Kim JT, Yu CW. Hazardous materials in buildings. Indoor Built Environ. 2014; 23: 44-61.

6. Wahlström M, Teittinen $T$, Kaartinen $T$, van Cauwenberghe L. Hazardous substances in construction products and materials: PARADE. Best practices for pre-demolition audits ensuring high quality raw materials. Brussels: EIT RawMaterials; 2019.

7. Wahlström M, zu Castell-Rüdenhausen M, Hradil P, Hauge-Smith K, Oberender A, Ahlm M, et al. Improving quality of construction \& demolition waste: Requirements for pre-demolition audit. Copenhagen: Nordic Council of Ministers; 2019.

8. Franzblau A, Demond AH, Sayler SK, D'Arcy H, Neitzel RL. Asbestos-containing materials in abandoned residential dwellings in Detroit. Sci Total Environ. 2020; 714: 136580.

9. Wilk E, Krówczyńska M, Zagajewski B. Modelling the spatial distribution of asbestos-cement products in Poland with the use of the random forest algorithm. Sustainability. 2019; 11: 4355.

10. Govorko $\mathrm{MH}$, Fritschi L, White J, Reid A. Identifying asbestos-containing materials in homes: Design and development of the ACM check mobile phone app. JMIR Form Res. 2017; 1: e7.

11. Govorko MH, Fritschi L, Reid A. Accuracy of a mobile app to identify suspect asbestos-containing material in Australian residential settings. J Occup Environ Hyg. 2018; 15: 598-606.

12. Govorko M, Fritschi L, Reid A. Using a mobile phone app to identify and assess remaining stocks of in situ asbestos in Australian residential settings. Int J Environ Res Public Health. 2019; 16: 4922.

13. Krówczyńska M, Raczko E, Staniszewska N, Wilk E. Asbestos-cement roofing identification using remote sensing and convolutional neural networks (CNNs). Remote Sens. 2020; 12: 408.

14. Adedeji $\mathrm{O}$, Wang $\mathrm{Z}$. Intelligent waste classification system using deep learning convolutional neural network. Procedia Manuf. 2019; 35: 607-612.

15. Bonifazi G, Capobianco G, Serranti S. Hyperspectral imaging and hierarchical PLS-DA applied to asbestos recognition in construction and demolition waste. Appl Sci. 2019; 9: 4587.

16. Chen C. Science mapping: A systematic review of the literature. J Data Inf Sci. 2017; 2: 1-40.

17. Moher D, Liberati A, Tetzlaff J, Altman DG, Altman D, Antes G, et al. Preferred reporting items for systematic reviews and meta-analyses: The PRISMA statement. PLoS Med. 2009; 6: e1000097.

18. Merriam-Webster. Ratio of a geometric progression [Internet]. Springfield, MA: MerriamWebster; 2021 [cited 2021 March 11th]. Available from: https://www.merriamwebster.com/dictionary/ratio\%20of\%20a\%20geometric\%20progression.

19. Laurie Kazan-Allen. Chronology of asbestos bans and restrictions [Internet]. International Ban Asbestos Secretariat; 2020 [cited 2021 January 17th]. Available from: 


\section{http://www.ibasecretariat.org/chron ban list.php.}

20. Wikipedia. Geometric progression [Internet]. 2021 [cited 2021 March 9th]. Available from: https://en.wikipedia.org/wiki/Geometric progression.

21. Herrick RF, Stewart JH, Allen JG. Review of PCBs in US schools: A brief history, an estimate of the number of impacted schools, and an approach for evaluating indoor air samples. Environ Sci Pollut Res. 2016; 23: 1975-1985.

22. Burdett G, Cottrell S, Taylor C. Airborne fibre and asbestos concentrations in system built schools. In: Journal of physics: Conference series. Bristol: IOP Publishing; 2009. pp.012023.

23. Brown KW, Minegishi T, Cummiskey CC, Fragala MA, Hartman R, MacIntosh DL. PCB remediation in schools: A review. Environ Sci Pollut Res. 2016; 23: 1986-1997.

24. Paglietti F, Malinconico S, Molfetta VD, Bellagamba S, Damiani F, Gennari F, et al. Asbestos risk: From raw material to waste management: The Italian experience. Crit Rev Environ Sci Technol. 2012; 42: 1781-1861.

25. STHDA. MCA-multiple correspondence analysis in R: Essentials-articles-STHDA [Internet]. 2017 [cited 2021 January 17]. Available from: http://www.sthda.com/english/articles/31-principalcomponent-methods-in-r-practical-guide/114-mca-multiple-correspondence-analysis-in-ressentials/\#dimension-description.

26. Wikipedia. Multiple correspondence analysis [Internet]. 2021 [cited 2021 March 11th]. Available from: https://en.wikipedia.org/wiki/Multiple correspondence analysis.

27. Garfield E. Historiographic mapping of knowledge domains literature. J Inf Sci. 2004; 30: 119145.

28. Bodar C, Spijker J, Lijzen J, Waaijers-van der Loop S, Luit R, Heugens E, et al. Risk management of hazardous substances in a circular economy. J Environ Manage. 2018; 212: 108-114.

29. Donovan S, Pickin J. An Australian stocks and flows model for asbestos. Waste Manag Res. 2016; 34: 1081-1088.

30. Kim YC, Hong WH. Optimal management program for asbestos containing building materials to be available in the event of a disaster. Waste Manage. 2017; 64: 272-285.

31. Mecharnia T, Chibout Khelifa L, Pernelle N, Hamdi F. An approach toward a prediction of the presence of asbestos in buildings based on incomplete temporal descriptions of marketed products. Proceedings of the 10th International Conference on Knowledge Capture; 2019 September 23rd; Marina del Rey, United States. New York: Association for Computing Machinery. pp.239-242.

32. Abriha D, Kovács Z, Ninsawat S, Bertalan L, Balázs B, Szabó S. Identification of roofing materials with discriminant function analysis and random forest classifiers on pan-sharpened WorldView2 imagery-a comparison. Hungarian Geogr Bull. 2018; 67: 375-392.

33. Gibril MB, Shafri HZ, Hamedianfar A. New semi-automated mapping of asbestos cement roofs using rule-based object-based image analysis and Taguchi optimization technique from WorldView-2 images. Int J Remote Sens. 2017; 38: 467-491.

34. Bonifazi G, Capobianco G, Serranti S. Asbestos containing materials detection and classification by the use of hyperspectral imaging. J Hazard Mater. 2018; 344: 981-993.

35. Rashidi A, Sigari MH, Maghiar M, Citrin D. An analogy between various machine-learning techniques for detecting construction materials in digital images. KSCE J Civ Eng. 2016; 20: 11781188.

36. Kuritcyn $P$, Anding $K$, Linß E, Latyev SM. Increasing the safety in recycling of construction and 
demolition waste by using supervised machine learning. In: Journal of physics: Conference series. Bristol: IOP Publishing; 2015. pp.012035.

37. Anding K, Garten D, Linß E. Application of intelligent image processing in the construction material industry. ACTA IMEKO. 2013; 2: 61-73.

38. Anding $K$, Linß E, Träger $H$, Rückwardt $M$, Göpfert A. Optical identification of construction and demolition waste by using image processing and machine learning methods. Proceedings of the Joint International IMEKO TC1+ TC7+ TC13 symposium and the Intelligent quality measurements-theory, education and training, in conjunction with the 56th IWK, IImenau University of Technology and the 11th SpectroNet Collaboration Forum; 2011 August 31stSeptember 2nd; Jena, Germany. Ilmenau: Technische Universität IImenau. pp.126-132.

39. Lewis M. Incompatible trends-hazardous chemical usage in building products poses challenges for functional circular construction. In: IOP conference series: Earth and environmental science. Bristol: IOP Publishing; 2019. pp.012046.

40. Armstrong B, Driscoll T. Mesothelioma in Australia: Cresting the third wave. Public Health Res Pract. 2016; 26: e2621614.

41. Lee RJ, Van Orden DR, Dunmyre GR. Interlaboratory evaluation of the breakup of asbestoscontaining dust particles by ultrasonic agitation. Environ Sci Technol. 1996; 30: 3010-3015.

42. Peto J, Matthews FE, Hodgson JT, Jones JR. Continuing increase in mesothelioma mortality in Britain. Lancet. 1995; 345: 535-539.

43. Ameille J, Brochard P, Brechot JM, Pascano T, Cherin A, Raix A, et al. Pleural thickening: A comparison of oblique chest radiographs and high-resolution computed tomography in subjects exposed to low levels of asbestos pollution. Int Arch Occup Environ Health. 1993; 64: 545-548.

44. Oliver LC, Sprince NL, Greene R. Asbestos-related disease in public school custodians. Am J Ind Med. 1991; 19: 303-316.

45. Kim SY, Kim YC, Kim Y, Hong WH. Predicting the mortality from asbestos-related diseases based on the amount of asbestos used and the effects of slate buildings in Korea. Sci Total Environ. 2016; 542: 1-11.

46. Frassy F, Candiani G, Rusmini M, Maianti P, Marchesi A, Nodari FR, et al. Mapping asbestoscement roofing with hyperspectral remote sensing over a large mountain region of the Italian Western Alps. Sensors. 2014; 14: 15900-15913.

47. Fiumi L, Campopiano A, Casciardi S, Ramires D. Method validation for the identification of asbestos-cement roofing. Appl Geomat. 2012; 4: 55-64.

48. Marinaccio A, Binazzi A, Cauzillo G, Cavone D, De Zotti R, Ferrante P, et al. Analysis of latency time and its determinants in asbestos related malignant mesothelioma cases of the Italian register. Eur J Cancer. 2007; 43: 2722-2728.

49. Fiumi L. Evaluation of MIVIS hyperspectral data for mapping covering materials. Proceedings of the IEEE/ISPRS Joint Workshop on Remote Sensing and Data Fusion over Urban Areas, DFUA 2001; 2002 August 6th; Rome, Italy. Piscataway Township: Institute of Electrical and Electronics Engineers Inc. pp.324-327.

50. Neumann V, Günther S, Müller KM, Fischer M. Malignant mesothelioma-German mesothelioma register 1987-1999. Int Arch Occup Environ Health. 2001; 74: 383-395.

51. Bourdès V, Boffetta P, Pisani P. Environmental exposure to asbestos and risk of pleural mesothelioma: Review and meta-analysis. Eur J Epidemiol. 2000; 16: 411-417.

52. Lange JH, Lange PR, Reinhard TK, Thomulka KW. A study of personal and area airborne asbestos 
concentrations during asbestos abatement: A statistical evaluation of fibre concentration data. Ann Occup Hyg. 1996; 40: 449-466.

53. Frederiksen M, Meyer HW, Ebbeh øj NE, Gunnarsen L. Polychlorinated biphenyls (PCBs) in indoor air originating from sealants in contaminated and uncontaminated apartments within the same housing estate. Chemosphere. 2012; 89: 473-479.

54. Robson M, Melymuk L, Csiszar SA, Giang A, Diamond ML, Helm PA. Continuing sources of PCBs: The significance of building sealants. Environ Int. 2010; 36: 506-513.

55. Diamond ML, Melymuk L, Csiszar SA, Robson M. Estimation of PCB stocks, emissions, and urban fate: Will our policies reduce concentrations and exposure? Environ Sci Technol. 2010; 44: 27772783.

56. Rudel RA, Seryak LM, Brody JG. PCB-containing wood floor finish is a likely source of elevated PCBs in residents' blood, household air and dust: A case study of exposure. Environ Health. 2008; 7: 2.

57. Herrick RF, Lefkowitz DJ, Weymouth GA. Soil contamination from PCB-containing buildings. Environ Health Perspect. 2007; 115: 173-175.

58. Broding HC, Schettgen T, Göen T, Angerer J, Drexler H. Development and verification of a toxicokinetic model of polychlorinated biphenyl elimination in persons working in a contaminated building. Chemosphere. 2007; 68: 1427-1434.

59. Kohler M, Tremp J, Zennegg M, Seiler C, Minder-Kohler S, Beck M, et al. Joint sealants: An overlooked diffuse source of polychlorinated biphenyls in buildings. Environ Sci Technol. 2005; 39: 1967-1973.

60. Priha E, Hellman S, Sorvari J. PCB contamination from polysulphide sealants in residential areasexposure and risk assessment. Chemosphere. 2005; 59: 537-543.

61. Kohler M, Zennegg M, Waeber R. Coplanar polychlorinated biphenyls (PCB) in indoor air. Environ Sci Technol. 2002; 36: 4735-4740.

62. Frederiksen M, Andersen HV, Haug LS, Thomsen C, Broadwell SL, Egsmose EL, et al. PCB in serum and hand wipes from exposed residents living in contaminated high-rise apartment buildings and a reference group. Int J Hyg Environ Health. 2020; 224: 113430.

63. Kolarik B, Frederiksen M, Meyer HW, Ebbehøj NE, Gunnarsen LB. Investigation of the importance of tertiary contamination, temperature and human behaviour on $\mathrm{PCB}$ concentrations in indoor air. Indoor Built Environ. 2016; 25: 229-241.

64. Liu X, Guo Z, Krebs KA, Greenwell DJ, Roache NF, Stinson RA, et al. Laboratory study of PCB transport from primary sources to building materials. Indoor Built Environ. 2016; 25: 635-650.

65. Liu X, Guo Z, Krebs KA, Stinson RA, Nardin JA, Pope RH, et al. Chamber study of PCB emissions from caulking materials and light ballasts. Chemosphere. 2015; 137: 115-121.

66. Lehmann GM, Christensen K, Maddaloni M, Phillips LJ. Evaluating health risks from inhaled polychlorinated biphenyls: Research needs for addressing uncertainty. Environ Health Perspect. 2015; 123: 109-113.

67. Meyer HW, Frederiksen M, Göen T, Ebbehøj NE, Gunnarsen L, Brauer C, et al. Plasma polychlorinated biphenyls in residents of 91 PCB-contaminated and 108 non-contaminated dwellings-an exposure study. Int J Hyg Environ Health. 2013; 216: 755-762.

68. Maclntosh DL, Minegishi T, Fragala MA, Allen JG, Coghlan KM, Stewart JH, et al. Mitigation of building-related polychlorinated biphenyls in indoor air of a school. Environ Health. 2012; 11: 24. 
69. Schettgen T, Alt A, Preim D, Keller D, Kraus T. Biological monitoring of indoor-exposure to dioxinlike and non-dioxin-like polychlorinated biphenyls (PCB) in a public building. Toxicol Lett. 2012; 213: 116-121.

70. Bloise A, Miriello D. Multi-analytical approach for identifying asbestos minerals in situ. Geosciences. 2018; 8: 133.

71. Bergmans J, Dierckx P, Broos K. Semi-selective demolition: Current demolition practices in Flanders. In: HISER conference. Delft: The Netherlands; 2017.

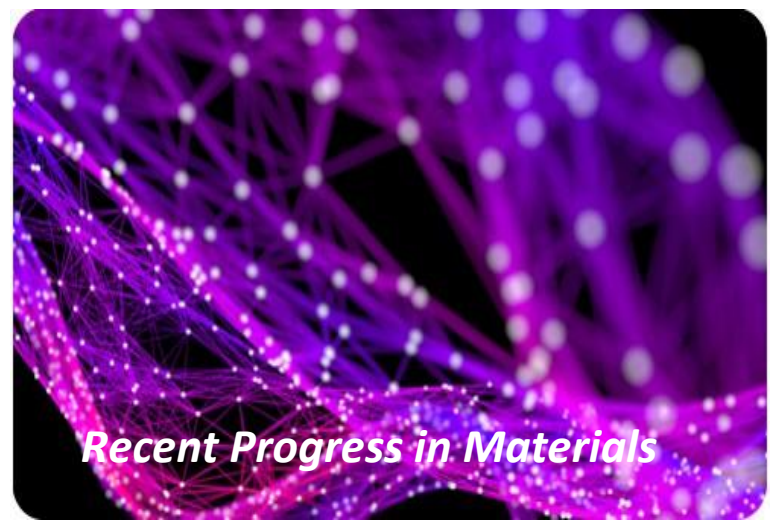

Enjoy Recent Progress in Materials by:

1. Submitting a manuscript

2. Joining in volunteer reviewer bank

3. Joining Editorial Board

4. Guest editing a special issue

For more details, please visit:

http://www.lidsen.com/journals/rpm 